\title{
Physiological Effects of Exogenous Melatonin on Leaves of Kiwifruit Seedlings under Drought Stress
}

\author{
Hui Xia ${ }^{1, a}$, Xiaojing Huang ${ }^{1,2, b}$, Jin Wang ${ }^{1,2, c}$, Xiulan Lv ${ }^{1,2, d}$ and Dong Liang ${ }^{1,2, e, *}$ \\ ${ }^{1}$ College of Horticulture, Sichuan Agricultural University, Chengdu, Sichuan, China \\ ${ }^{2}$ Institute of Pomology and Olericulture, Sichuan Agricultural University, Chengdu, Sichuan, China \\ asusanxia_2001@163.com, b1301621709@qq.com, c251040278@qq.com, d1933063229@qq.com, \\ eliangeast@sina.com \\ * corresponding author
}

\section{Keywords: Kiwifruit; Drought stress; Melatonin}

Abstract. Melatonin is a small molecular substance widely found in higher plants and can improve plant resistance to stress ability. To investigate the effects of melatonin on the response mechanism and drought resistance kiwifruit seedlings, one-year-old seedlings of kiwifruit and root irrigation method were used to study the physiological effects of exogenous melatonin on kiwifruit seedlings under drought stress. The results showed that the relative electrical conductivity, soluble sugar content, proline content and total flavanol (TFAC) content of kiwifruit seedlings in the drought-treated group are increasing and the soluble protein content decreases first and then increases when compared with the control group $(\mathrm{CK})$; root irrigation MT solution can effectively reduce the relative conductivity of kiwifruit seedlings leaves, and significantly increase soluble sugar, soluble protein, proline and TFAC content. The results show that MT can effectively reduce the harm of drought stress, improve kiwifruit adapt to drought stress environment.

\section{Introduction}

Kiwifruit is the 20th century wild fruit artificial cultivation of the most successful one of the four fruit [1]. China's kiwifruit cultivation area and output rank are first in the world. In china, kiwifruit grows in large span from north to south, and requires high environmental conditions, especially soil moisture $[2,3]$. The roots of kiwifruit are fleshy roots and the root porosity is only $1.0-2.7 \%$, so the root system is extremely sensitive to soil moisture [4]. Therefore, soil moisture has become one of the important factors restricting the scale of kiwifruit cultivation.

MT (melatonin), as a broad spectrum of physiological regulators, ubiquitous in animals and plants and micro-organisms [5,6]. In plants, MT has strong antioxidant activity by scavenging reactive oxygen species and free radicals, which can improve the resistance of plants to drought, heavy metals, high temperature, salt, and other adverse conditions [7-10]. At present, there are few reports on the resistance of exogenous MT to kiwifruit under drought stress. In this experiment, we studied the physiological effects of exogenous MT on the seedlings of kiwifruit under drought stress, and provided the theoretical basis for the rational use of melatonin to solve the drought problem in kiwifruit cultivation.

\section{Materials and Methods}

Materials and Treatment. The seeds of kiwifruit were placed at $4^{\circ} \mathrm{Cand} 60-70 \%$ relative humidity for 60 days in winter to relieve dormancy. After variable temperature treatment at $4^{\circ} \mathrm{C} 10 \mathrm{~h}, 25^{\circ} \mathrm{C} 14 \mathrm{~h}$ for one week, seeds were placed at $25^{\circ} \mathrm{Cfor} 10-15$ days to germinate. When the seedlings at 2-day intervals, we transferred the seedlings to plastic pots filled with mixed soil consisting of peat substrate, pulverized coconut shell and perlite. Then began watering the seedlings at 2-dintervals with $1 / 2$ Hoagland's nutrient solution from the two-true-leaf stage ( $\mathrm{pH}$ adjusted to $6.5 \pm 0.1$ with diluted $\mathrm{HCl}$ or $\mathrm{NaOH})$. 
When grown up with 9-10 true leaves, we started treatments as follows: (1) Control (CK), normal watering during the test period; (2) drought treatment (DR): Watered prior to treatment and then not watering $9 \mathrm{~d}$ seedlings were well-watered for 8 days, subsequently irrigation was withheld for up to 9 days; (3) melatonin and drought treatment (MTDR): seedlings were pretreated with $100 \mu \mathrm{M}$ melatonin solution for 4 times, then no irrigation for up to 9 days. Their middle leaves (from five to eight per plant) were sampled after $0,3,6$, and $9 \mathrm{~d}$ of drought treatment. All collected tissues were immediately frozen in liquid nitrogen and stored at $-80^{\circ} \mathrm{C}$.

Enzymes Assay and Indicator Determintion. Relative electric conductivity determination method adopted Xiong et al. [11]; MDA and proline content determination method adopted Li et al. [12], determination of TFAC using the p-DMACA method [13].

Data Analysis. Analysis of variance was performed using the SPSS software (SPSS, Inc., Chicago, IL, USA). Each treatment was replicated three times. Significant differences were detected using Duncan's test at the $\mathrm{P}<0.05$ level.

\section{Results and Analysis}

Relative Conductivity. Under drought stress, the relative electrical conductivity of leaves of kiwifruit seedling was increased and peaked at 9d, when compared with CK increased by $120.52 \%$. However, when pretreated with MT, the relative electrical conductivity of the kiwifruit leaves was higher than that of the CK group, but the change was not significant; when Compared with the DR group, the relative conductivity of the kiwifruit leaves decreased significantly, especially at 9d, 1.7 times lower than that of the drought group (Fig.1).

\section{$\square C K$ QDR $\mathbb{M M T D R}$}

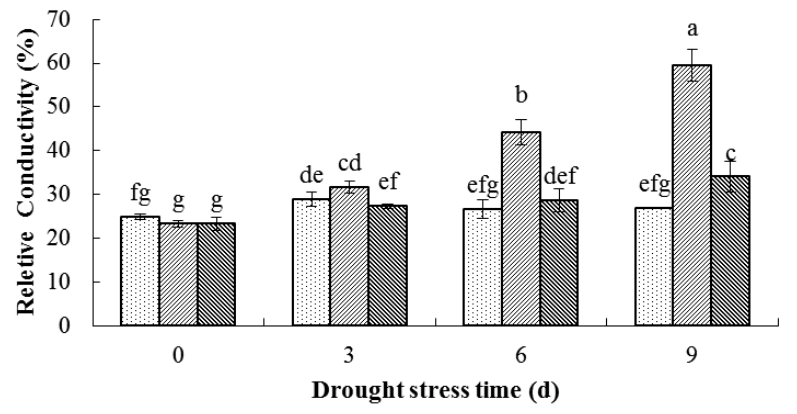

Fig.1 Effects of Exogenous Melatonin on Relative Conductivity of Kiwi Seedling Leaves under Drought Stress. Note: Data with the different letters indicate the difference is significant $(\mathrm{P}<0.05)$.

Soluble sugar, Soluble Protein and Proline Content. Under drought stress, the soluble sugar content of Kiwifruit leaves did not change significantly at 0-6d, then increased significantly and peaked at $9 \mathrm{~d}$ with $2.70 \%$, when compared with CK increased by 3.18 times. However, After the pretreatment of melatonin, the soluble sugar content in the leaves was slowly increasing and peaked at 9d with $1.76 \%$; when compared with DR group, the soluble sugar content in the leaves was higher at 0-6 days, but at 9d below DR group (Fig.2A). The soluble protein content in the leaves of DR and MTDR treatments was decreased first and drop to the minimum at $6 \mathrm{~d}$ with $4.05 \mathrm{mg} \cdot \mathrm{g}^{-1} \mathrm{FW}$ and $4.50 \mathrm{mg} \cdot \mathrm{g}^{-1} \mathrm{FW}$, compared with $\mathrm{CK}$ decreased by 0.66 and 0.74 times, and then increased towards. Moreover, the soluble protein content in MTDR was significant higher than those in DR all over the time (Fig.2B). The proline content of leaves of kiwifruit seedling was increased and peaked at $9 \mathrm{~d}$ with $152.55 \mu \mathrm{g} \cdot \mathrm{g}^{-1} \mathrm{FW}$, compared with $\mathrm{CK}$ increased by 2.4 times. when pretreated with MT, the proline content of leaves of kiwifruit seedling was increased and peaked at 9d, which was $114.12 \mu \mathrm{g}^{-1} \mathrm{FW}$. When compared with DR, the content of proline in leaves was low in the DR group, but the difference between the two groups was not statistically significant (Fig.2C). 

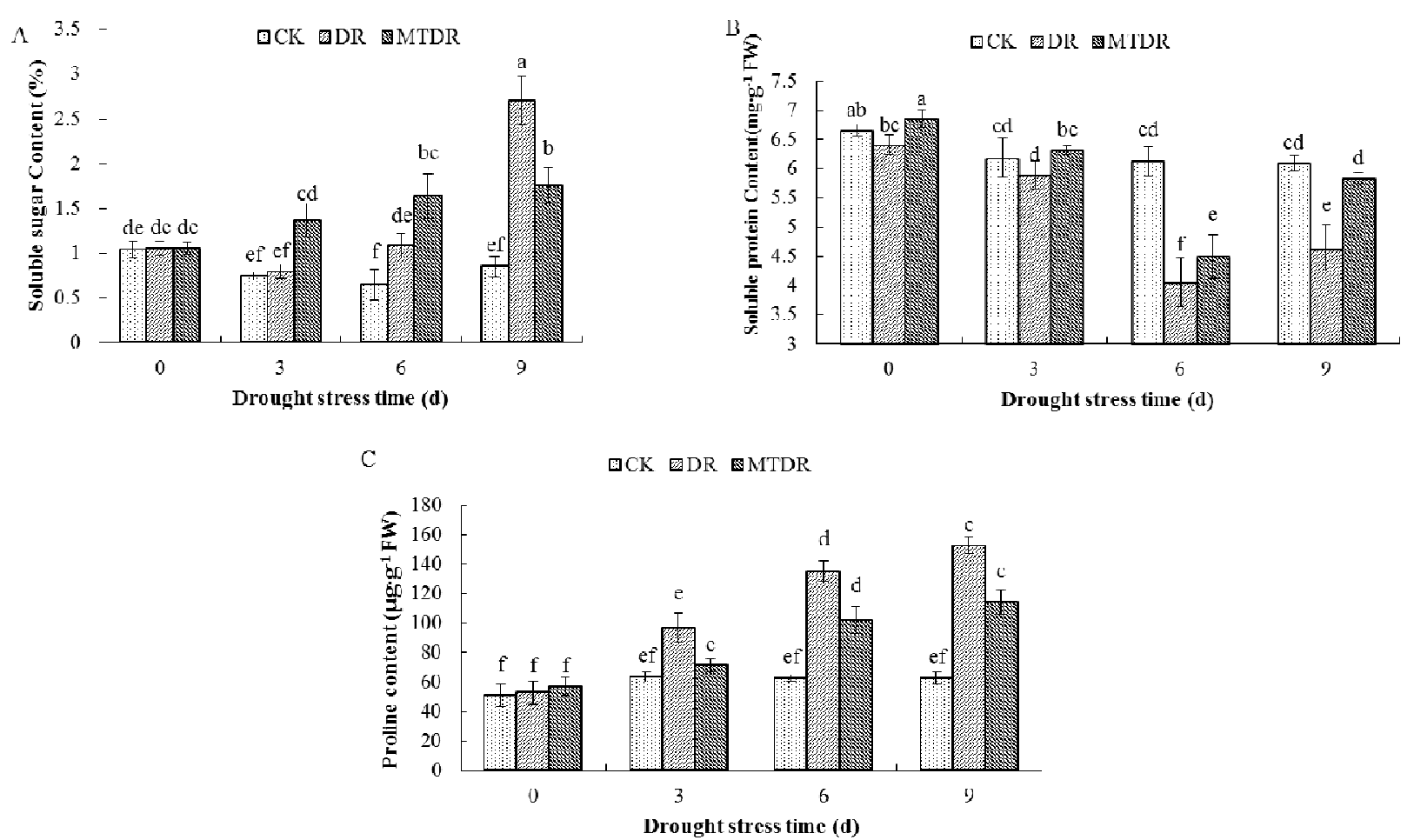

Fig.2 Effects of Exogenous Melatonin on Contents of Soluble Sugar (A), Soluble Protein (B) and Proline (C) in Leaves of Kiwifruit Seedlings. Note: Data with the different letters indicate the difference is significant $(\mathrm{P}<0.05)$.

TFAC Content. The difference of TFAC content between DR and MTDR groups was not significant, when compared with $\mathrm{CK}$, the TFAC content of DR and MTDR groups increased significantly from 0 to 3 days and peaked at $3 \mathrm{~d}$ with $0.59 \mathrm{mg} \mathrm{CE} \cdot \mathrm{g}^{-1} \mathrm{FW}$ and $0.60 \mathrm{mg} \mathrm{CE} \cdot \mathrm{g}^{-1} \mathrm{FW}$, then decreased to the level of CK.

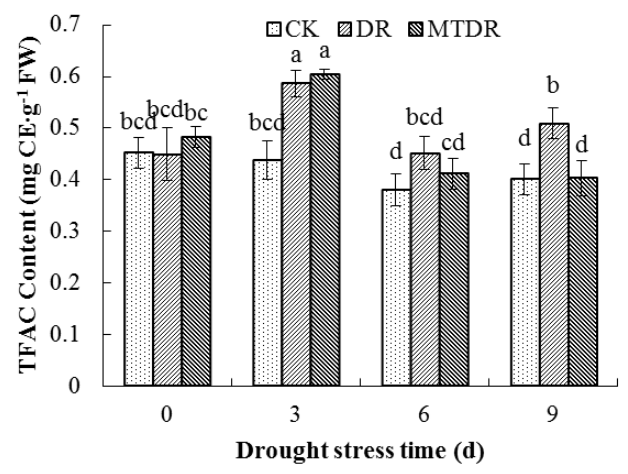

Fig.3 Effects of Exogenous Melatonin on TFAC Content in Kiwi Seedling Leaves. Note: Data with the different letters indicate the difference is significant $(\mathrm{P}<0.05)$.

\section{Conclusion}

Proline, soluble sugar and soluble protein are osmotic regulators, which are the protective system of plant in the process of long-term evolution, and the change of relative conductivity content is related to the drought resistance of plants [14]. Total flavanol belong to phenols, studies have shown that secondary metabolites such as phenols may be involved in the removal of reactive oxygen species as a second line of defense under stress [15]. In this study, the relative electrical conductivity of kiwifruit seedlings increased under drought stress, indicating that the plants were damaged to some extent, and the contents of proline and soluble sugar in leaves increased, indicating that the plants opened self-protection mechanism during this period. After the pretreatment of melatonin, the relative conductivity and proline content decreased significantly, the TFAC content increased, the content of soluble sugar and soluble protein in 0-6 days higher than GR, indicating that melatonin can relieve 
membrane lipid peroxidation, protect the stability of cell membrane, To further improve the resistance of kiwi fruit in arid environment.

\section{Acknowledgements}

This work was supported by fund of the Science and Technology Department of Sichuan Province (2016NZ0105).

\section{References}

[1] I.J. Warrington and G.C. Weston: Ray Richards Publisher. Forum Vol. 183-204(1990), p. 186

[2] Y.H. Peng and W.C. Zhang: Chinese Fruit Trees. Forum Vol. 44-45(1 995), p.44 (in Chinese)

[3] A.R. Freguson: Acta Horticulturae. Forum Vol. 603-653(1990), p.290

[4] C. Zhang: Zhejiang Normal University. 2013. (in Chinese)

[5] Y. Zhao, D.H. Wang and X.Y. Zhao: Journal of Northwest Botanical Sciences. Forum Vol. 196-205(2014), p.200 (in Chinese)

[6] S.J. Murch, A.R. Alan and J. Cao: Journal of Pineal Research. Forum Vol. 277(2009), p.277

[7] N. Zhang, B. Zhao and H.J. Zhang: Journal of Pineal Research. Forum Vol. 15-23(2013), P.18

[8] J. Limson, T. Nyokong and S. Daya: Journal of Pineal Research. Forum Vol. 15-21(1998), p.19

[9] Y. Byeon and K. Back: Journal of Pineal Research. Forum Vol. 189-195(2014), p.192

[10]C. Li, P. Wang and Z. Wei: Journal of Pineal Research. Forum Vol. 298-306(2012), p.303

[11]Q.Z. Xu and B.R. Huang: Crop Science. Forum Vol. 1363-1368(2000), p.1366

[12]Hesheng Li: Principles and Techniques of Plant Physiological and Biochemical Experiment. Higher Education Press. 2000 (in Chinese)

[13]G.Y. Li, G. Tanner and P. Larkin: Journal of the Science of Food \& Agriculture. Forum Vol. 89-101(1996)

[14]Q. Sun, N.H. Cai and S. Chen: Journal of Southwest Forestry University. Forum Vol. 18-22(2016), p.21 (in Chinese)

[15]A. Fini, L. Guidi and F. Ferrini: Journal of Plant Physiology. Forum Vol. 929-939(2012), p.936 